\title{
EVIDENCE FOR ELEVATED X-RAY EMISSION IN LOCAL LYMAN BREAK GALAXY ANALOGS
}

\author{
Antara R. Basu-Zych ${ }^{1}$, Bret D. Lehmer ${ }^{1,2}$, Ann E. Hornschemeier ${ }^{1,2}$, Thiago S. Gonçalves ${ }^{3}$, Tassos Fragos ${ }^{4}$, \\ Timothy M. Heckman ${ }^{2}$, Roderik A. Overzier ${ }^{5,6}$, ANDrew F. PtaK ${ }^{1,2}$, AND David Schiminovich ${ }^{7}$ \\ ${ }^{1}$ NASA Goddard Space Flight Center, Code 662, Greenbelt, MD 20771, USA; antara.r.basu-zych@ @asa.gov \\ ${ }^{2}$ Department of Physics and Astronomy, The Johns Hopkins University, 3400 North Charles Street, Baltimore, MD 21218, USA \\ ${ }^{3}$ Observatório do Valongo, Universidade Federal do Rio de Janeiro, Ladeira Pedro Antonio 43, Saúde, Rio de Janeiro-RJ, CEP 22240-060, Brazil \\ ${ }^{4}$ Harvard-Smithsonian Center for Astrophysics, 60 Garden Street, Cambridge, MA 02138, USA \\ ${ }^{5}$ Department of Astronomy, University of Texas at Austin, 1 University Station C1400, Austin, TX 78712, USA \\ ${ }^{6}$ Observatório Nacional, Rua José Cristino 77, São Cristóvão, Rio de Janeiro-RJ, CEP 20921-400, Brazil \\ ${ }^{7}$ Department of Astronomy, Columbia University, 550 West 120th Street, New York, NY 10027, USA \\ Received 2013 June 3; accepted 2013 July 18; published 2013 August 26
}

\begin{abstract}
Our knowledge of how X-ray emission scales with star formation at the earliest times in the universe relies on studies of very distant Lyman break galaxies (LBGs). In this paper, we study the relationship between the 2-10 keV X-ray luminosity $\left(L_{X}\right)$, assumed to originate from X-ray binaries (XRBs), and star formation rate (SFR) in ultraviolet (UV) selected $z<0.1$ Lyman break analogs (LBAs). We present Chandra observations for four new Galaxy Evolution Explorer selected LBAs. Including previously studied LBAs, Haro 11 and VV 114, we find that LBAs demonstrate $L_{X} /$ SFR ratios that are elevated by $\sim 1.5 \sigma$ compared to local galaxies, similar to the ratios found for stacked LBGs in the early universe $(z>2)$. Unlike some of the composite LBAs studied previously, we show that these LBAs are unlikely to harbor active galactic nuclei, based on their optical and X-ray spectra and the spatial distribution of the X-rays in three spatially extended cases. Instead, we expect that high-mass X-ray binaries (HMXBs) dominate the X-ray emission in these galaxies, based on their high specific SFRs (sSFRs $\equiv \mathrm{SFR} / M_{\star} \geqslant 10^{-9} \mathrm{yr}^{-1}$ ), which suggest the prevalence of young stellar populations. Since both UV-selected populations (LBGs and LBAs) have lower dust attenuations and metallicities compared to similar samples of more typical local galaxies, we investigate the effects of dust extinction and metallicity on the $L_{X} /$ SFR for the broader population of galaxies with high SSFRs $\left(>10^{-10} \mathrm{yr}^{-1}\right)$. The estimated dust extinctions (corresponding to column densities of $N_{\mathrm{H}}<10^{22} \mathrm{~cm}^{-2}$ ) are expected to have insignificant effects on observed $L_{X} /$ SFR ratio for the majority of galaxy samples. We find that the observed relationship between $L_{\mathrm{X}} / \mathrm{SFR}$ and metallicity appears consistent with theoretical expectations from XRB population synthesis models. Therefore, we conclude that lower metallicities, related to more luminous HMXBs such as ultraluminous X-ray sources, drive the elevated $L_{X} /$ SFR observed in our sample of $z<0.1$ LBAs. The relatively metal-poor, active mode of star formation in LBAs and distant $z>2$ LBGs may yield higher total HMXB luminosity than found in typical galaxies in the local universe.
\end{abstract}

Key words: galaxies: evolution - galaxies: high-redshift - galaxies: starburst - X-rays: binaries

Online-only material: color figures

\section{INTRODUCTION}

$\mathrm{X}$-ray binaries (XRBs) dominate the $2-10 \mathrm{keV}$ emission in normal galaxies that do not harbor powerful active galactic nuclei (AGNs). In normal star-forming galaxies, high-mass $\mathrm{X}$-ray binaries (HMXBs), associated with young $(<100 \mathrm{Myr})$ stellar populations, drive the observed correlation between X-ray luminosity and star formation rate (SFR; e.g., Ranalli et al. 2003; Grimm et al. 2003; Bauer et al. 2002; Hornschemeier et al. 2005; Lehmer et al. 2008; Mineo et al. 2012a).

Recently, Basu-Zych et al. (2013) found evidence for a mild increase in the mean 2-10 keV luminosity per SFR $\left(L_{X} / \mathrm{SFR}\right)$ with redshift for star-forming galaxy populations spanning the majority of cosmic history $(z=0-5)$. This result is consistent with XRB population synthesis models, which predict that an increase in $L_{X} / \mathrm{SFR}$ with redshift is expected in response to the declining metallicities of galaxies (Fragos et al. 2013a).

Currently, the hard X-ray emission in high redshift $(z>2)$ galaxies, such as Lyman break galaxies (LBGs), can only be studied in an average sense by "stacking" the X-ray counts from large numbers of objects within the deepest Chandra surveys (Brandt et al. 2001; Nandra et al. 2002; Seibert et al. 2002; Lehmer et al. 2005; Cowie et al. 2012; Zheng et al. 2012; Zinn et al. 2012; Basu-Zych et al. 2013). The few LBGs detected individually in the $\mathrm{X}$-ray band at $z \approx 3$ are dominated by AGNs rather than star formation related activity (Brandt et al. 2001; Nandra et al. 2002; Lehmer et al. 2005; Laird et al. 2006). By contrast, LBGs that are not powered by AGNs are extremely $\mathrm{X}$-ray faint sources. Observing these sources on an individual basis would require Chandra exposures of $\approx 8-20 \mathrm{yr}$, well beyond practical observational limits.

Local $(D \leqslant 200 \mathrm{Mpc} ; z \leqslant 0.02)$ starbursts have served as a training set for studying the detailed properties likely to be present in distant LBGs. However, local galaxies differ greatly in the mode of star formation from LBGs. For instance, LBGs suffer only modest dust extinction in the ultraviolet (UV; e.g., Webb et al. 2003), whereas local starbursts with comparable SFRs are very dusty with only a few percent of the UV emission escaping (e.g., Heckman et al. 1998; Adelberger \& Steidel 2000). Furthermore, LBGs in the early universe $(z>2)$ formed their stars from more pristine lower-metallicity gas than local galaxies (Erb et al. 2006), potentially producing more luminous HMXBs per SFR (Mapelli et al. 2009; Zampieri \& Roberts 2009; Fragos et al. 2013b).

While the star formation conditions present in LBGs (i.e., high SFR, dust- and metal-poor) were commonplace at high 

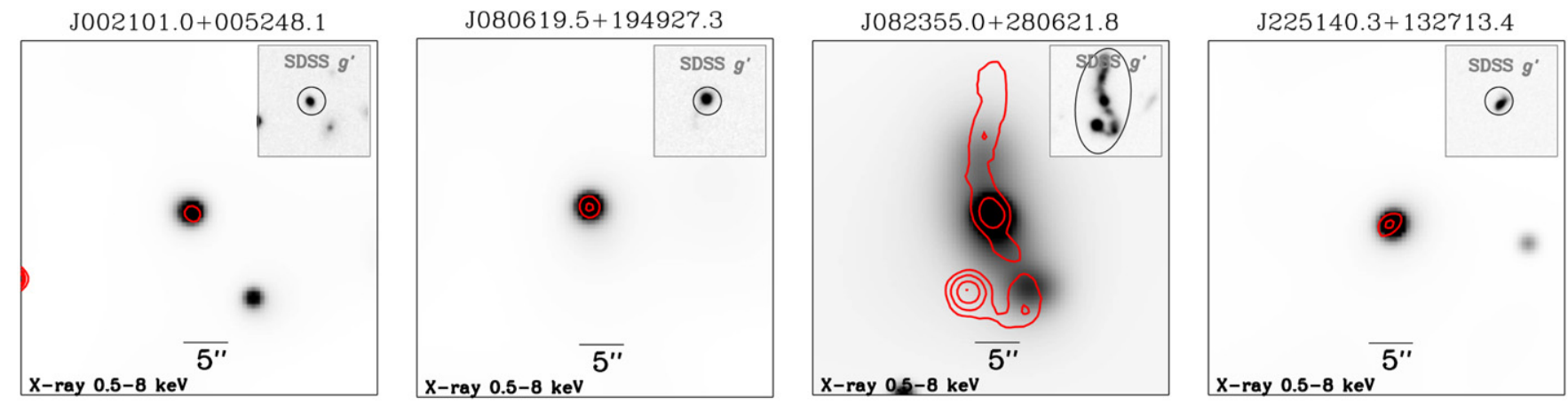

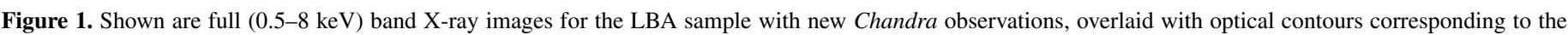

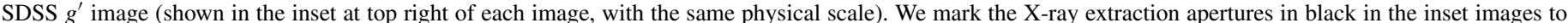

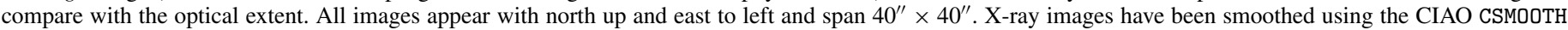

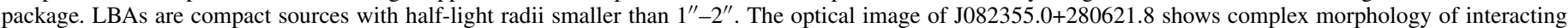

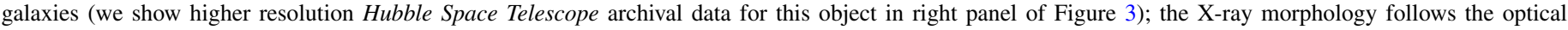
morphology. The bright optical source in the southeast is a foreground star, based on optical spectra taken with the Apache Point Observatory $3.5 \mathrm{~m}$ telescope.

(A color version of this figure is available in the online journal.)

redshift, they appear relatively rare in the local universe. Galaxy Evolution Explorer (GALEX) all-sky surveys permit the discovery of UV-selected galaxies that are in this currently rare mode. These local $(z<0.3)$ Lyman break analogs (LBAs) are selected to have far-UV (FUV) properties consistent with $z>2$ LBGs: e.g., high SFRs $\left(\sim 10-30 M_{\odot} \mathrm{yr}^{-1}\right)$, little dust attenuation $\left(\log L_{\mathrm{IR}} / L_{\mathrm{UV}} \lesssim 1\right)$, and relatively low gas-phase metallicities $(12+\log [\mathrm{O} / \mathrm{H}]<8.5)$. Based on their similarities with LBGs and near proximities, LBAs provide the ideal opportunity to study X-ray emission in individual LBG-like galaxies. A sample of six $z \sim 0.2$ LBAs, targeted for having optical emission-line properties that are intermediate between starbursts and obscured (type 2) AGNs, have been studied by Jia et al. (2011) using XMM and their results suggest the likely presence of type 2 AGNs. However, with the exception of these "composite" LBAs and two other individual cases (VV 114 and Haro 11; Grimes et al. 2006, 2007), X-ray studies of galaxies have not targeted the LBA population comprehensively.

In this paper, we remedy our limited knowledge of the $\mathrm{X}$-ray properties of LBAs and present Chandra observations of six star-formation-dominated LBAs. We define the LBA sample in Section 2.1 and compare our sample of six LBAs to other larger samples of star-forming galaxies, described in Section 2.2. We find that LBAs have elevated $L_{X}$ per SFR, compared to these other samples, and offer our physical interpretation for this result in Section 3. We investigate possible physical causes, including the possible presence of AGNs (Section 3.2), the effects of metallicity (Section 4.2), and dust extinction (Section 4.3). We discuss the implications of our results on the high redshift universe in Section 5. Section 6 summarizes our results and outlines future plans. Throughout this paper we assume a Kroupa (2001) initial mass function (IMF), converting accordingly when comparing with the literature, and adopt a standard $\Lambda$ CDM cosmology $\left(H_{0}=70 \mathrm{~km} \mathrm{~s}^{-1} \mathrm{Mpc}^{-1}\right.$, $\left.\Omega_{M}=0.3, \Omega_{\Lambda}=0.7\right)$.

\section{DATA AND ANALYSIS}

\subsection{The LBA Sample}

The launch of GALEXenabled rest-frame UV-selection of relatively nearby $(z<0.3)$ galaxies in order to discover potential local LBG analogs. Heckman et al. (2005) introduced such a sample of UV luminous galaxies (UVLGs) with FUV $\left(\lambda \sim 1550 \AA\right.$ ) luminosities, $L_{\mathrm{FUV}}>2 \times 10^{10} L_{\odot}$. This selection was further refined by adding an FUV surface brightness criterion $\left(I_{\mathrm{FUV}}>10^{9} L_{\odot} \mathrm{kpc}^{-2}\right.$; Hoopes et al. 2007) in order to select "super-compact UVLGs" with similar global properties as LBGs. Since this time, several studies have established these galaxies, henceforth called LBAs, as excellent analogs to LBGs (Basu-Zych et al. 2007, 2009; Overzier et al. 2008, 2009, 2010, 2011; Gonçalves et al. 2010; Heckman et al. 2011). LBAs are also notably different from other local star-forming galaxies, the former having lower dust attenuations per SFR (Basu-Zych et al. 2007; Overzier et al. 2011) and metallicities per stellar mass (Overzier et al. 2009) than the latter. LBAs are physically compact (half-light diameters $\approx 1-2 \mathrm{kpc}$; see contours and inset image in Figure 1), often disturbed systems whose Sloan Digital Sky Survey (SDSS) optical spectra indicate that they are starbursts (see Figure 4).

We selected the sample of LBAs to be studied by Chandra by cross-matching the SDSS DR7 spectroscopic and photometric data with the GALEX GR3 data to find galaxies with $L_{\mathrm{FUV}}>2 \times 10^{10} L_{\odot}$ and $I_{\mathrm{FUV}}>10^{9} L_{\odot} \mathrm{kpc}^{-2}$. In this process, we used the SDSS $u$-band half-light radius $\left(r_{u, 50}\right)$, based on the SDSS DR7 expRad_u catalog value, to approximate the FUV surface brightness $\left(I_{\mathrm{FUV}} \equiv L_{\mathrm{FUV}} / \pi r_{u, 50}^{2}\right.$; see the Appendix in Overzier et al. 2008 for further discussion). To determine $L_{\mathrm{FUV}}$, we $k$-corrected the observed GALEX magnitudes using version 4 of the $\mathrm{k}$-correct (Blanton et al. 2003) IDL code with the sevenband GALEX+SDSS (FUV, NUV, $u^{\prime}, g^{\prime}, r^{\prime}, i^{\prime}, z^{\prime}$ ) magnitudes. These steps for selecting LBAs are identical to the ones taken by Hoopes et al. (2007) in the original selection of LBAs, with the sole exception that we started with updated catalogs, resulting in 301 LBAs. For this pilot program, we targeted the four closest $(z<0.1)$ and most UV-luminous LBAs with Chandra, discussed in the following Section 2.1.1. In addition, we include in our LBA sample two other Chandra-observed galaxies that are considered good analogs of LBGs: Haro 11 and VV 114. Haro 11 satisfies the aforementioned LBA selection criteria; VV 114 has an FUV luminosity of $2.5 \times 10^{10} L_{\odot}$ and FUV surface brightness of $\sim 7.8 \times 10^{8} L_{\odot} \mathrm{kpc}^{-2}$, comparable to LBAs and LBGs.

\subsubsection{X-Ray Measurements of LBAs}

Chandra observed four SDSS+GALEX-selected LBAs in Cycle 12, using the Advanced CCD Imaging Spectrometer (ACIS)-S camera in the very faint observing mode. We used the 
Table 1

Lyman Break Analogs: Summary of Chandra X-Ray Observations

\begin{tabular}{|c|c|c|c|c|c|c|c|c|}
\hline \multirow[t]{2}{*}{ ID } & \multirow{2}{*}{$\begin{array}{c}D \\
(\mathrm{Mpc})\end{array}$} & \multirow[t]{2}{*}{ ObsID } & \multirow{2}{*}{$\begin{array}{c}\text { Exposure } \\
(\mathrm{ks})\end{array}$} & \multirow{2}{*}{$\begin{array}{c}\text { Aperture } \\
\left({ }^{\prime \prime}\right)\end{array}$} & \multicolumn{2}{|c|}{ Net Counts } & \multirow{2}{*}{$\begin{array}{c}\text { Flux }(2-10 \mathrm{keV})^{\mathrm{a}} \\
\left(10^{-15} \mathrm{erg} \mathrm{s}^{-1} \mathrm{~cm}^{-2}\right)\end{array}$} & \multirow[t]{2}{*}{$\phi^{2-8} / \phi^{0.5-2}$} \\
\hline & & & & & $0.5-2 \mathrm{keV}$ & $2-10 \mathrm{keV}$ & & \\
\hline J002101.0+005248.1 & 452 & 13014 & 19.4 & 5 & $27.0_{-5.3}^{+6.4}$ & $6.4_{-2.8}^{+4.0}$ & $7.0_{-3.1}^{+4.4}$ & $0.2_{-0.1}^{+0.2}$ \\
\hline J080619.5+194927.3 & 315 & 13015 & 20.0 & 5 & $24.0_{-5.0}^{+6.1}$ & $9.3_{-3.3}^{+4.4}$ & $9.9_{-3.5}^{+4.7}$ & $0.4_{-0.2}^{+0.3}$ \\
\hline J082355.0+280621.8 & 210 & 13012 & 9.0 & $10 \times 20^{\mathrm{b}}$ & $42.9_{-6.8}^{+7.8}$ & $13.0_{-4.3}^{+5.4}$ & $31.1_{-1.0}^{+1.3}$ & $0.3_{-0.1}^{+0.2}$ \\
\hline $\mathrm{J} 225140.3+132713.4$ & 279 & 13013 & 19.8 & 5 & $42.8_{-6.6}^{+7.7}$ & $9.0_{-3.3}^{+4.4}$ & $9.7_{-3.5}^{+4.8}$ & $0.2_{-0.1}^{+0.2}$ \\
\hline
\end{tabular}

Notes.

a $2-10 \mathrm{keV}$ flux assuming simple power law spectrum with $N_{\mathrm{H}}=3 \times 10^{20} \mathrm{~cm}^{-2}$ (average Galactic extinction for the sample, with variations in this value between different galaxies contributing to $\sim 3 \%$ uncertainty) and $\Gamma=1.9$.

b We used a slightly tilted (position angle of -7.6) elliptical aperture, based on the optical structure, to account for any extended emission in this galaxy.

Chandra Interactive Analysis of Observations (CIAO) package version 4.4.1 with Chandra Calibration Database version 4.5.3 for our analysis. Table 1 summarizes the Chandra observations for the LBAs. The exposure times (see Column 3) ranged from $\sim 9$ to $20 \mathrm{ks}$. We produced exposure maps following the procedure described in Hornschemeier et al. (2001, Section 3.2). Briefly, these maps were normalized to the effective exposures of sources located at the aim points and include the effects from vignetting, gaps between the CCDs, bad column and pixel filtering, and the spatially dependent degradation of the ACIS optical blocking filter. A photon index of $\Gamma=1.4$ was assumed in creating the exposure maps.

For our range of off-axis angles, the $90 \%$ encircled energy fraction radius is $\sim 1.2^{\prime \prime}$. However, we typically extracted counts within a $5^{\prime \prime}$ aperture to sufficiently include the extent of the optical emission, except in the case of J082355.0+280621.8 where we used a $10 \times 20^{\prime \prime}$ (radius) elliptical aperture to account for the larger extent of emission in this galaxy (see the third panel in Figure 1). The insets in Figure 1 show the extraction aperture compared to the optical extent of the galaxy. To determine the background counts, we measured the counts within a $15^{\prime \prime}$ radial aperture at 50 random locations between $\sim 30^{\prime \prime}-60^{\prime \prime}$ away from our target to properly sample the background statistics such that the error in the background contributes negligibly to the calculation of the net counts. In order to ensure that this random background region did not coincide with another source, we repeated this process (i.e., selected a new background region randomly) if any pixels within the background region exceeded 1.5 times the mean of the background distribution. The total counts from this background region were scaled by the ratios of the source to background aperture areas to give the net counts. We determined fluxes using PIMMS, assuming a power law with $\Gamma=1.9$, since it was the power law index that best fit our combined LBA spectrum (see Section 3.2.2), and Galactic extinction, $N_{\mathrm{H}}=3 \times 10^{20} \mathrm{~cm}^{-2}$, which is the average value for these four LBAs, using the COLDEN Galactic neutral hydrogen density calculator. The $N_{\mathrm{H}}$ values range from $1-5 \times 10^{20} \mathrm{~cm}^{-2}$, corresponding to negligible $(\sim 3 \%)$ error on the flux calculations. Based on the SDSS spectroscopically determined redshift, we apply a $(1+z)^{\Gamma-2.0}=(1+z)^{-0.3}$ $k$-correction to the $2-10 \mathrm{keV}$ fluxes to calculate $2-10 \mathrm{keV}$ X-ray luminosities (provided in last column of Table 2).

Grimes et al. (2006, 2007) have conducted detailed X-ray studies on VV 114 and Haro 11 and we refer to these papers for the information presented in Columns 2-4 and 9 of Table 2. We also compare our sample with a sample of $z \sim 0.2 \mathrm{LBAs}$, whose X-ray emission was studied by Jia et al. (2011, henceforth J11) using $X M M$.

\subsection{Comparison Samples}

For comparison with the LBAs, we include other samples of "normal" (not containing AGNs) local star-forming galaxies, studied using Chandra. These local star-forming galaxies include 24 nearby spiral and irregular galaxies studied by Colbert et al. (2004) and 22 additional star-forming galaxies from Mineo et al. (2012a, hereafter referred to as M12; 7 of the original 29 galaxies are also in the Colbert et al. 2004 sample). In both studies, the galaxies are sufficiently nearby to spatially resolve the $\mathrm{XRB}$ population; these authors extracted the X-ray luminosities from XRBs (specifically, HMXBs in the M12 sample) separate from the rest of the X-ray emission in these galaxies.

In addition, our comparison samples include luminous and ultraluminous infrared galaxies (LIRGs and ULIRGs), galaxies which have infrared (IR) luminosities exceeding $10^{11} L_{\odot}$ and $10^{12} L_{\odot}$, respectively. These IR-selected galaxies are dusty, high SFR galaxies, whose $\mathrm{X}$-ray properties have been explored in a number of X-ray studies (Lehmer et al. 2010; Iwasawa et al. 2011; Symeonidis et al. 2011). We include 13 LIRGs/ULIRGs from Lehmer et al. (2010, hereafter L10), after eliminating 4 likely AGNs from their sample of 17, and 29 LIRGs/ULIRGs from the Great Observatories All-Sky LIRG Survey (GOALS; Iwasawa et al. 2011), excluding 15 classified AGNs from their total sample of 44. While many of the LIRGs/ULIRGs are spatially resolved with Chandra, the X-ray luminosities refer to galaxy-wide 2-10 keV emission.

We obtain 2-10 keV X-ray luminosities $\left(L_{X}\right)$ from the references above, converting luminosities from other bands to the 2-10 keV band in some cases (e.g., $0.3-8 \mathrm{keV}$, Colbert et al. 2004; 0.5-8 keV, M12). In cases where $L_{X}$ was derived using a power law with $\Gamma \neq 1.9$ or using other X-ray bands (e.g., $\Gamma=1.8$, Colbert et al. 2004; $\Gamma=2.0, \mathrm{M} 12$ ), we converted to $\Gamma=1.9$ for the sake of consistency. We note that $L_{\mathrm{X}}$ values from both LIRG/ULIRG samples (L10; Iwasawa et al. 2011) are calculated from fits to the individual galaxy spectra and include a hot-gas component $(k T \lesssim 0.8 \mathrm{keV})$ plus power law $(\Gamma=1-3)$. Since these are likely to be more accurate than using an assumed power law, we use their quoted values of $L_{X}$.

Therefore, the complete sample of comparison galaxies includes 88 galaxies.

\subsection{UV and IR Properties: SFR and IRX}

While UV radiation traces recent star formation, it is also easily absorbed by dust and reradiated as IR radiation. Therefore a proper accounting of the total SFR includes both UV+IR, as described in the following relation from Bell et al. (2005):

$$
\operatorname{SFR}\left(M_{\odot} \mathrm{yr}^{-1}\right)=9.8 \times 10^{-11}\left(L_{\mathrm{IR}}+3.3 L_{\mathrm{UV}}\right),
$$


Table 2

Lyman Break Analogs: Derived Quantities

\begin{tabular}{|c|c|c|c|c|c|c|c|c|c|}
\hline $\begin{array}{l}\text { ID } \\
\text { (1) }\end{array}$ & $\begin{array}{l}\text { R.A. } \\
\text { (deg) } \\
(2)\end{array}$ & $\begin{array}{c}\text { Decl. } \\
\text { (deg) } \\
(3)\end{array}$ & $\begin{array}{l}z^{\mathrm{a}} \\
(4)\end{array}$ & $\begin{array}{c}r_{u, 50} \\
(\mathrm{kpc}) \\
(5)\end{array}$ & $\begin{array}{c}\log M_{\star}^{\mathrm{b}} \\
\left(M_{\odot}\right) \\
(6)\end{array}$ & $\begin{array}{c}\mathrm{SFR}^{\mathrm{c}} \\
\left(M_{\odot} \mathrm{yr}^{-1}\right) \\
(7)\end{array}$ & $\begin{array}{c}\text { IRX } \\
(8)\end{array}$ & $\begin{array}{c}12+\log (\mathrm{O} / \mathrm{H})^{\mathrm{d}} \\
(9)\end{array}$ & $\begin{array}{c}L_{\mathrm{X}}^{\mathrm{e}} \\
\left(10^{40} \mathrm{erg} \mathrm{s}^{-1}\right)\end{array}$ \\
\hline J002101.0+005248.1 & 5.254 & 0.880 & 0.098 & 0.84 & 9.75 & 23.70 & 0.65 & 8.19 & $17_{-7}^{+10}$ \\
\hline J080619.5+194927.3 & 121.581 & 19.824 & 0.070 & 0.66 & 9.52 & 15.16 & 0.96 & 8.15 & $12_{-4}^{+6}$ \\
\hline J082355.0+280621.8 & 125.979 & 28.106 & 0.047 & 0.44 & 9.46 & 17.11 & 0.88 & 8.23 & $16_{-5}^{+7}$ \\
\hline $\mathrm{J} 225140.3+132713.4$ & 342.918 & 13.454 & 0.062 & 1.54 & 9.39 & 8.65 & 0.53 & 8.15 & $9_{-3}^{+4}$ \\
\hline VV114 & 16.946 & -17.507 & 0.020 & $2.3^{\mathrm{f}}$ & 10.65 & 37.82 & 1.0 & 8.45 & $24^{\mathrm{f}}$ \\
\hline Haro11 & 9.219 & -33.555 & 0.020 & $1.1^{\mathrm{f}}$ & 9.84 & 10.88 & 0.52 & 8.33 & $12^{\mathrm{f}}$ \\
\hline
\end{tabular}

Notes.

${ }^{a}$ Spectroscopic redshifts taken from the SDSS DR7 spectroscopic catalog, with $\Delta z /(1+z)<0.008$.

b Stellar mass uncertainties can be as large as 0.4 dex (Zibetti et al. 2009).

${ }^{c}$ UV+IR SFR.

d We used gas-phase metallicities from the SDSS DR7 MPA/JHU VAC (the SDSS DR7 MPA/JHU value-added catalogs are available from http://www.mpa-garching.mpg.de/SDSS/), which were determined using the technique outlined by Tremonti et al. (2004).

e $2-10 \mathrm{keV}$ X-ray luminosity.

${ }^{f}$ Other LBAs studied by Grimes et al. (2006, 2007).

where $L_{\mathrm{IR}}$ and $L_{\mathrm{UV}}$ correspond to the total IR $(8-1000 \mu \mathrm{m})$ and UV ( $v L_{v}$ at $2800 \AA$ ) luminosities, respectively, in units of solar luminosity. The factor of 3.3 is the typical correction factor that brings $v L_{v}$ (at $2800 \AA$ ) to the integrated $L_{\mathrm{UV}}$. All of the LBAs have been observed by the Wide-field Infrared Survey Explorer (WISE; Wright et al. 2010) and detected in the $22 \mu \mathrm{m}$ band. We estimated $L_{\mathrm{IR}}$ by converting the monochromatic $22 \mu \mathrm{m}$ luminosity to the total $8-1000 \mu \mathrm{m}$ luminosity by using the Chary \& Elbaz (2001) IR spectral energy distribution template. SFRs have been calculated using the $L_{\mathrm{UV}}$ determined from GALEX data, ${ }^{8}$ the $L_{\mathrm{IR}}$ determined from WISE data, ${ }^{9}$ and Equation (1). The amount of dust attenuation in the UV can be approximated by the IR-excess (IRX), defined as

$$
\mathrm{IRX}=\log \left(L_{\mathrm{IR}} / L_{\mathrm{UV}}\right) .
$$

SFRs and IRX values for the LBAs are presented in Columns 7 and 8 in Table 2. We estimate SFRs and IRX in a consistent way for the comparison samples using the available values of $L_{\mathrm{IR}}$ and $L_{\mathrm{UV}}$ from the related papers (see Section 2.2; given in Howell et al. 2010 for the GOALS sample of LIRG/ULIRGs and obtained from S. Mineo 2013, private communication for the M12 sample).

\subsection{Stellar Mass and Metallicity}

We estimated stellar masses using Two Micron All Sky Survey (2MASS) $K_{s}$ magnitudes and following the relations given by Table 7 of Bell et al. (2003), which are calculated for different choices of optical/NIR colors. The colors used in the stellar mass measurements are $\operatorname{SDSS} u^{\prime}-z^{\prime}, B-K$ and $B-V$ (where $B$ and $V$ photometric data come from the Third Reference Catalog of Bright Galaxies (RC3); de Vaucouleurs et al. 1991) colors for the LBAs, Colbert et al. (2004) star-forming and L10 LIRG samples, respectively. Howell et al. (2010) use 2MASS $K_{s}$ magnitudes and IRAC $3.6 \mu \mathrm{m}$ to estimate $M_{\star}$ in the GOALS (U)LIRGs, which agrees well (slightly lower by $\sim 4 \%$, see

\footnotetext{
8 GALEX data release GR3 catalog description can be found here: http://galex.stsci.edu/GR6/?page=ddfaq (see also Morrissey et al. 2007). We measured $L_{\mathrm{UV}}$ using the NUV $(\lambda=2500 \AA)$ data.

9 Details describing the WISE All-Sky catalog and $22 \mu \mathrm{m}$ flux measurements can be found at http://wise2.ipac.caltech.edu/docs/release/allsky/expsup/ and in the following papers: Wright et al. (2010) and Jarrett et al. (2011).
}

L10) with our method. M12 estimate $M_{\star}$ from 2MASS $K_{s}$ magnitudes, applying the relation given by Equation (2) of Gilfanov (2004), which is based on models from Bell \& de Jong (2001), and we adjust these values to our adopted IMF (Kroupa). We note that Zibetti et al. (2009) show that the stellar masses estimated using different methods can vary by $\sim 0.4$ dex, which does not affect any of the final results that we present in this paper.

We measure the metallicities using the method outlined in Pettini \& Pagel (2004), using the O III $\lambda 5007$ and $\mathrm{N}_{\text {II }} \lambda 6584$ emission-line ratios (referred to, hereafter, as the PP04 O3N2 method). Kewley \& Ellison (2008) compare different metallicity measurement techniques and find that different estimated gas-phase metallicities can differ systematically by 0.7 dex depending on the method. While calibration from various methods to an absolute metallicity value remains uncertain, relative uncertainties are minimized when using one consistent method and the PP04 method proves to be one of the most robust methods (Kewley \& Ellison 2008). Emission-line measurements are available for all 6 of the LBAs and 38 of the 88 comparison galaxies, which we henceforth refer to as the "partial comparison sample." Using published emission-line values (28 comparison star-forming galaxies, 10 LIRGs, Haro 11, and VV 114; Kim et al. 1995; Wu et al. 1998; Moustakas \& Kennicutt 2006; Bergvall \& Östlin 2002) and SDSS spectroscopic data (4 Chandra-observed LBAs and 1 LIRG) we determine gasphase metallicities.

The stellar masses and gas-phase metallicities for the LBAs are provided in Columns 6 and 9 (respectively) in Table 2.

\section{RESULTS}

\subsection{Elevated $L_{X} / S F R$ in $U V$-selected Galaxies}

We show the relation between the 2-10 keV X-ray luminosity and SFR ( $L_{X}$ versus SFR) for the LBAs compared to the full star-forming sample of 88 galaxies in Figure 2 (see legend for symbol descriptions). We also compare with results from stacked $z=1.5-4$ LBGs (Basu-Zych et al. 2013, open squares), where individually detected X-ray sources (potential AGNs) were eliminated in order to study the X-ray emission related to star formation. On average, the UV-selected samples (LBAs and the stacked $z=1.5-4$ LBGs) appear to have $L_{\mathrm{X}}$ per SFR 


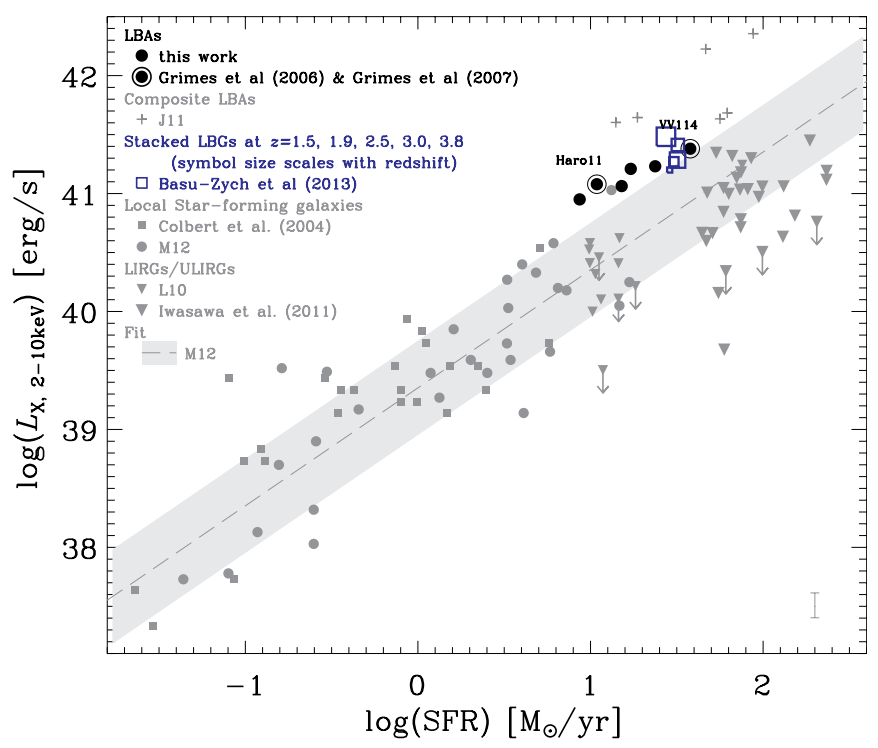

Figure 2. The LBAs (black points) and stacked LBGs (open squares; BasuZych et al. 2013) are significantly more X-ray luminous (in 2-10 keV band) per SFR compared to other galaxy populations: local galaxies (gray squares from Colbert et al. 2004 and gray points from M12) and LIRGs/ULIRGs (filled gray triangles from Iwasawa et al. 2011 and L10). The gray dashed line (and shaded region) shows the fit (and $1 \sigma$ scatter) based on the HMXB-dominated galaxies from M12, which we refer to as the local X-ray/SFR relation. We show the J11 sample of composite LBAs as crosses for comparison. The error bar in the lower right corner shows the level of uncertainty in $L_{\mathrm{X}}$ due to the choice of $\Gamma=1.9 \pm 0.2$.

(A color version of this figure is available in the online journal.)

elevated by a factor of $\sim 1.5 \sigma$ compared to the comparison sample, which is described by the dashed line and gray region (showing $1 \sigma$ ) based on the fit to $L_{\mathrm{X}} / \mathrm{SFR}$ from M12 (converted to the $2-10 \mathrm{keV}$ band, using $\Gamma=1.9)$ and given by

$$
\log L_{\mathrm{X}}(2-10 \mathrm{keV})=39.4( \pm 0.4) \log \mathrm{SFR} .
$$

The error bar shown in the lower right corner of Figure 2 describes the uncertainty attributed to our choice of $\Gamma=$ $1.9 \pm 0.2$, which accounts for the different assumed values for the power law index in the different samples (e.g., $\Gamma=2.0$, M12; 1.8, Colbert et al. 2004). We note that the uncertainty is $\sim 0.1$ dex, which does not change the result that the LBAs have significantly elevated $L_{\mathrm{X}}$ per SFR compared to the other samples. We also test whether biases are introduced from comparing samples that used different techniques for measuring $\mathrm{X}$-ray luminosities; comparing luminosities for galaxies in multiple samples (i.e., there are seven galaxies that are in M12 and Colbert et al. 2004), we find the data agree within $10 \%$ with no systematic differences. The likelihood that all six LBAs would randomly scatter this far from the local relation is $\sim 10^{-5}$ (based on $\chi^{2}$ ), almost certainly implying there is something fundamentally different about LBAs compared to typical normal galaxies. We note that there are some other galaxies which appear to have elevated $L_{X} / S F R$, which we discuss in Sections 4.1 and 4.2.

The J11 sample of $z \sim 0.2$ LBAs, having optical emissionline signatures between starbursts and obscured AGNs (see further discussion in Section 3.2.1), are shown in Figure 2 as crosses. Two of the J11 sources have $L_{\mathrm{X}} / \mathrm{SFR}$ that are extreme (factors $\sim 10-20$ above the relation), likely suggesting that AGNs power the X-ray emission in these sources; two other sources appear similarly elevated (by $\sim 1.5 \sigma$, or factor of $\sim 4$ ) as the LBAs in our sample.
Several studies have shown that $2-10 \mathrm{keV}$ emission in local galaxies correlates well with SFR (e.g., Ranalli et al. 2003; Persic \& Rephaeli 2007; Lehmer et al. 2010; Mineo et al. 2012a). The $2-10 \mathrm{keV}$ emission mainly originates from accreting XRBs (including ultraluminous X-ray sources (ULXs)). Although minimized by studying emission in the $2-10 \mathrm{keV}$ band, we note that a number of other sources can also contribute to X-ray emission in normal galaxies: supernovae and their remnants, and hot gas from starburst-driven winds and outflows (see, e.g., review by Fabbiano 1989; Strickland et al. 2000; Grimes et al. 2005; Li \& Wang 2013; Mineo et al. 2012b). Low-luminosity AGN activity may also contribute to the $2-10 \mathrm{keV}$ X-ray luminosity in normal galaxies. In the next section, we assess the likelihood that the presence of AGNs cause the elevated $L_{\mathrm{X}} /$ SFR observed in LBAs.

\subsection{Assessing Any Possible AGN Contribution}

Since AGNs also produce significant X-ray emission, we explore the possibility that hidden AGNs may cause $L_{X}$ values per SFR that are $\sim 1.5 \sigma$ higher in the LBAs. Benefiting from the high angular resolution capability of Chandra, three of the six LBAs in our sample, VV 114, Haro 11, and J082355.0+280621.8, appear to have extended X-ray emission (see Figure 3), and we can distinguish multiple $\mathrm{X}$-ray point sources, all of which are luminous enough to qualify as ULXs $\left(L_{X}>10^{40} \mathrm{erg} \mathrm{s}^{-1}\right)$ based on the limiting luminosity of the available data (e.g., ULXs have $L_{\mathrm{X}} \gtrsim 10^{41} \mathrm{erg} \mathrm{s}^{-1}$ in the case of J082355.0+280621.8). Using the images for these three LBAs, we measure the flux in the nuclear regions, potentially associated with AGNs, compared to the entire galaxy and find that the nuclear regions contribute only $<15 \%$ to the $2-10 \mathrm{keV}$ emission in each case, immediately indicating that the elevated $L_{\mathrm{X}} / \mathrm{SFR}$ for those LBAs cannot be attributed to AGN activity.

While VV114 may harbor an obscured AGN (in the eastern component, VV114E), Grimes et al. (2007) conclude that star formation dominates the $2-10 \mathrm{keV}$ power in this galaxy. Based on Spitzer/IRS spectroscopic analysis of the [O IV] $\lambda 25.9 \mu \mathrm{m}$ and $[\mathrm{Ne} \mathrm{v}] \lambda 14.3 \mu \mathrm{m}$ emission features, Cormier et al. (2012) conclude that Haro 11 does not contain an AGN. To further test for the presence of AGNs in the four newly observed SDSS+GALEX-selected LBAs, we use optical spectral line diagnostics and X-ray spectral constraints (see below).

\subsubsection{Optical Spectral Line Diagnostics}

The Baldwin-Phillips-Terlevich (BPT; Baldwin et al. 1981) diagram separates galaxies whose optical emission is dominated by AGNs versus star formation using the ratios of forbidden lines (i.e., [N II] $\lambda 6584$, [O III] $\lambda$ 5007), which are excited mainly by AGNs, to nearby Balmer lines. In the left panel of Figure 4, we show the distribution of $>900,000$ sources from the SDSS DR7 catalog (shaded background). The solid gray line is based on a theoretical modeling of the upper limit that star-forming galaxies can occupy in this diagram by Kewley et al. (2001). Kauffmann et al. (2003) revise the classification criterion to further separate star-forming from composite galaxies. The LBAs in this study (black points) reside in the star-forming region of this diagram.

Using XMM, J11 studied a sub-sample of six $z \sim 0.2$ LBAs (shown as crosses in Figures 2, 4, and 5) that lie within or near the composite region of the BPT diagram. They also found that these galaxies are systematically offset with respect to the X-ray/SFR relation, having $L_{\mathrm{X}}=4-20 \times 10^{41} \mathrm{erg} \mathrm{s}^{-1}$ (see Figure 2). Based on comparing the X-ray luminosities with the far-IR and [O III] $\lambda 5007$ luminosities, J11 conclude 

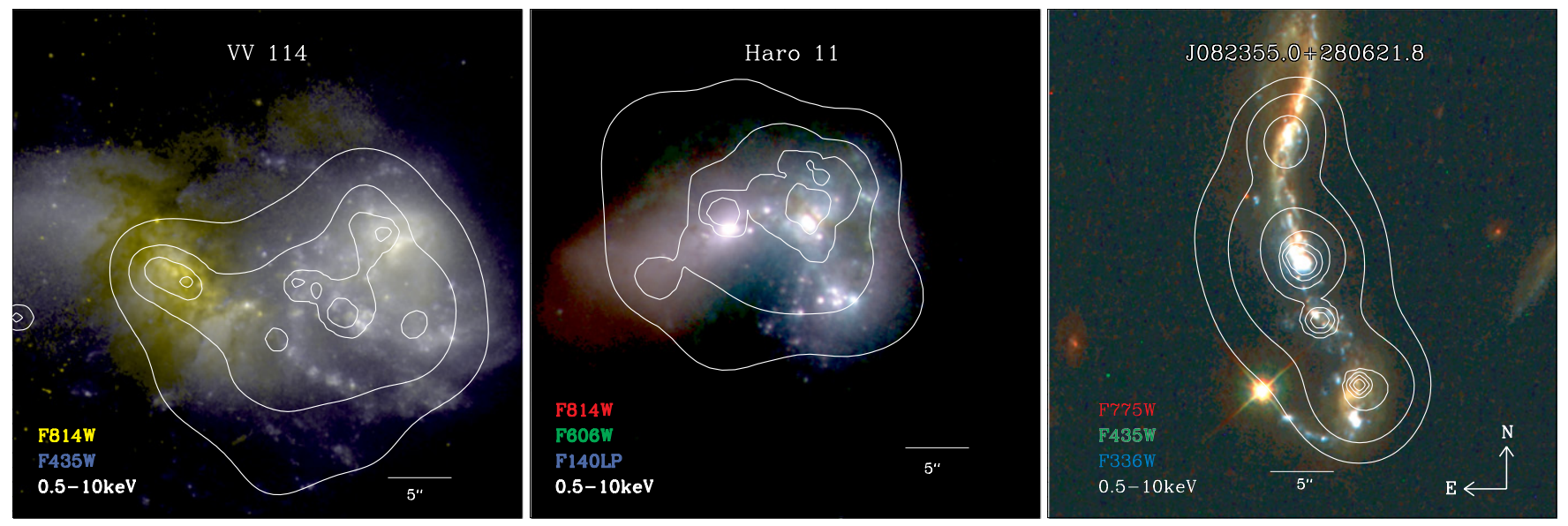

Figure 3. We present archival Hubble Space Telescope images of VV 114 (left; PI: A. Evans), Haro11 (center; PI: G. Östlin) and LBA J082355.0+280621.8 (right; PI: G. Östlin) with white contours showing the Chandra full-band $(0.5-10 \mathrm{keV})$ distribution, smoothed using CSMOOTH. We are able to resolve multiple X-ray sources with the Chandra data for these galaxies, which appear to host a higher number of ULXs than expected, based on the HMXBs in typical star-forming galaxies.

(A color version of this figure is available in the online journal.)
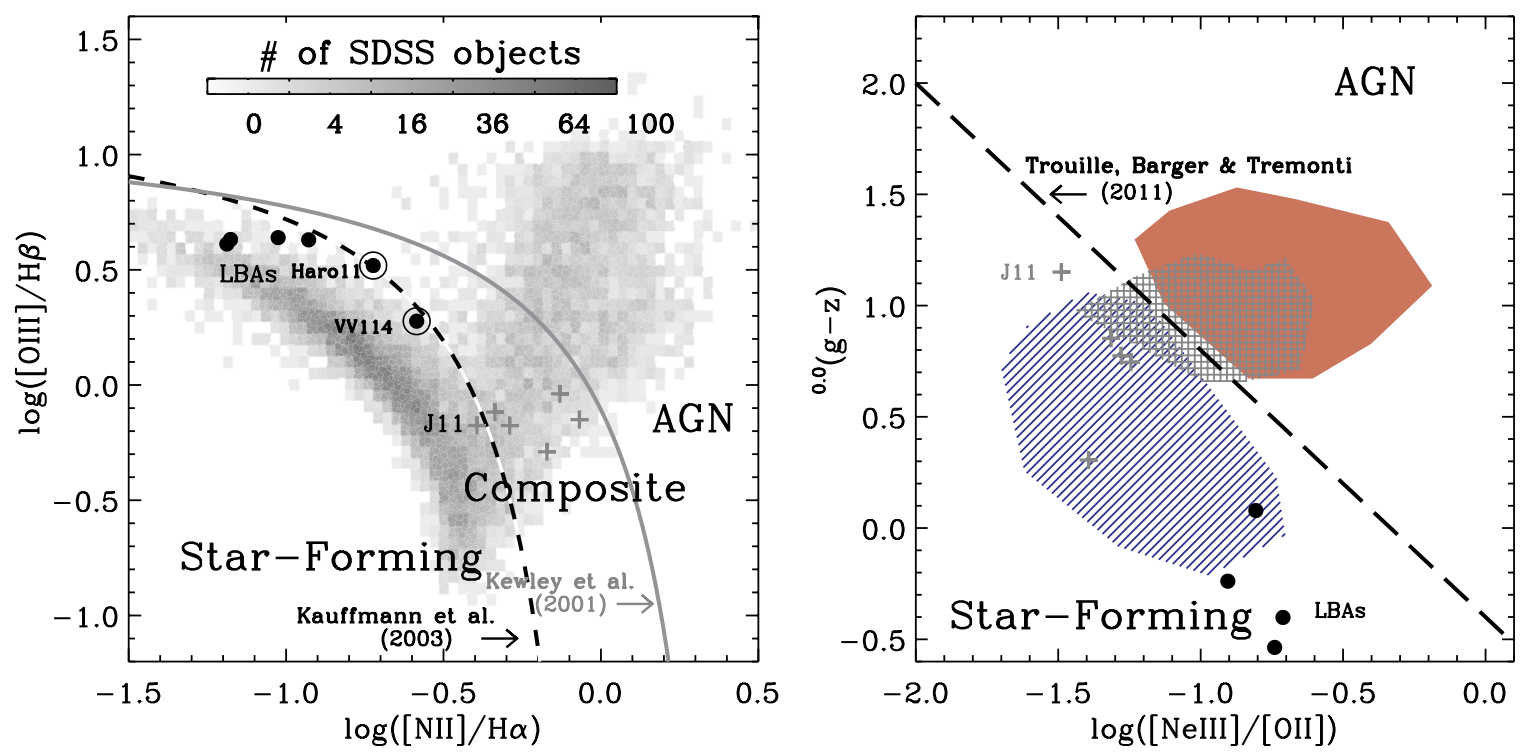

Figure 4. We use two different AGN vs. star formation diagnostics: BPT diagram (left) and TBT diagram (right; Trouille et al. 2011). Left: the full SDSS DR7 galaxy sample is shown (background data) with $z<0.1 \mathrm{LBAs}$ (black points). These emission-line ratios are determined after subtracting off template galaxy spectra and are very sensitive to even low-level AGN activity. The two lines in the diagram indicate the theoretical upper limit for star formation (gray upper solid line; Kewley et al. 2001) and the lower line below which AGNs are not expected (Kauffmann et al. 2003; black dashed line). Right: the TBT diagnostic separate AGN (above dashed line) from star-forming galaxies. The LBA sample falls well within the "star-forming" space for both diagnostics. We have also used velocity widths to screen against AGNs. The J11 sample of "composite LBAs" is shown as crosses.

(A color version of this figure is available in the online journal.)

that type 2 AGNs are likely present within their sample. One of the composite LBAs most offset in $L_{\mathrm{X}}$ was also shown to host a compact radio source using very long baseline interferometry (VLBI). The compact source was interpreted as an AGN having a small fraction of the total radio flux of the LBA (Alexandroff et al. 2012). Several other LBA composites were not detected at VLBI resolution, showing that the overall (i.e., large-scale) radio emission of LBAs is dominated by star formation.

Motivated by the study by Hornschemeier et al. (2005) which showed that using the BPT diagram alone may still include AGNs (e.g., narrow-line Seyfert 1 galaxies), we confirmed that our sample of LBAs had Balmer line widths that were consistent with those of the forbidden lines (within $3 \sigma$ compared to the distribution of the entire SDSS DR7 population).
Another emission-line test has been proposed by Trouille et al. (2011), referred hereafter by the TBT test (shown on right panel of Figure 4), which uses rest-frame $g-z$ color and the ratio of [Ne III] $\lambda 3869$ to [O II] $\lambda \lambda 3726,3729$ to separate AGNs (upper region) from star-forming galaxies (below dashed line). Trouille et al. (2011) compare their method with the BPT diagnostic; the solid red, dashed blue, and cross-hatched gray regions in Figure 4 (right) correspond to the BPT-determined AGN, starforming, and composite classifications, respectively. According to this classification, the LBAs reside in the star-forming region of the TBT diagram.

The TBT method is good for selecting sources that might be X-ray detected. In their analysis, Trouille et al. (2011) find that X-ray sources with $L_{\mathrm{X}}>10^{42} \mathrm{erg} \mathrm{s}^{-1}$ appear in the top region, while few luminous $\mathrm{X}$-ray sources fall below the dashed 


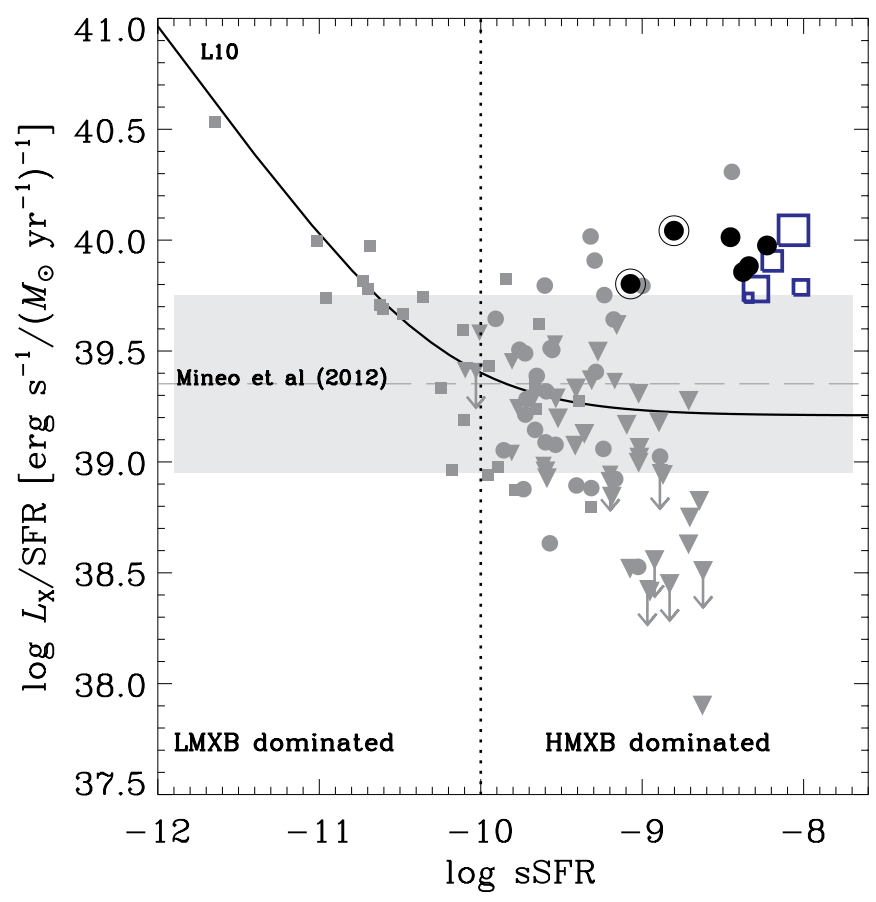

Figure 5. We compare $L_{X} /$ SFR vs. sSFR for LBAs (black points), LBGs (blue squares, symbol size increasing with sample redshift from $z=1.5-4$ ), and other star-forming galaxies (symbols are described in Figure 2). Specific SFRs (sSFRs) separate galaxies whose X-ray luminosity is dominated by LMXBs (left of the dashed line) from those dominated by HMXBs (with sSFR $\left.>10^{-10} M_{\odot} \mathrm{yr}^{-1}\right)$. The solid black line shows the fit from L10, and the gray shaded region and dashed line show the HMXB-driven relation from M12. Similar to LIRGs/ULIRGs, LBAs have high sSFRs, and therefore $L_{X}$ in LBAs is expected to be dominated by HMXBs. However, LBAs appear to have elevated $L_{\mathrm{X}} / \mathrm{SFR}$, compared to other high sSFR galaxies (including the LIRGs and ULIRGs), potentially driven by their lower metallicities.

(A color version of this figure is available in the online journal.)

line. Moreover, they show that the TBT method is able to classify luminous, less luminous, and soft X-ray detected non-broad line AGN sources accurately, while $\sim 20 \%$ of X-ray detected AGNs are classified as star-forming galaxies. We note that the J11 "composite LBAs" (crosses), based on the BPT classification, were detected at $L_{\mathrm{X}} \sim 10^{42} \mathrm{erg} \mathrm{s}^{-1}$, yet fall in the star-forming region of the TBT classification.

Our sample of LBAs lies conservatively within the star-forming region of the TBT diagram, providing further support against AGNs. While using optical emission lines to detect AGNs is expected to be sensitive to even low-levels of AGN activity, this method may suffer from aperture dilution in some of the higher redshift sources or may miss heavily obscured AGNs.

\subsubsection{X-Ray Spectral Properties of LBAs}

We test for the presence of obscured AGNs, whose optical spectral signatures may be hidden due to attenuation by dust. Assuming a power law spectrum, the X-ray hardness ratio (defined here as the ratio of emission in the $2-10 \mathrm{keV}$ band to that in the $0.5-2 \mathrm{keV}$ band $(\mathrm{H} / \mathrm{S}))$ is affected by the X-ray absorption column, $N_{\mathrm{H}}$, and the slope of the power law, $\Gamma$. The hardness ratios are listed in Table 1, and range between 0.2-0.4, suggesting very soft $\mathrm{X}$-ray spectra $(\Gamma=2.8-3.3)$.

While we do not have enough counts to fit the X-ray spectra for individual LBAs, we can perform a joint fit to the four Chandra-observed LBAs to obtain an average spectral shape for the sample. Even combining the data from four sources does not provide the adequate number of counts to constrain a spectrum with multiple components and therefore we restrict our analysis to fitting a simple power law. For the $0.5-8 \mathrm{keV}$ band, we find that the best fit power law has $\Gamma_{0.5-8}=1.91 \pm 0.15$, consistent with XRB and ULX (Swartz et al. 2004) spectra and inconsistent with heavily obscured and unobscured low-luminosity AGNs with $L / L_{\text {Edd }} \lesssim 0.1$ (e.g., Shemmer et al. 2006).

Additionally, the X-ray emission in starburst galaxies may contain a significant hot gas component, which would contribute strongly to the soft band, as found in the other LBAs, Haro 11 (Grimes et al. 2007) and VV 114 (Grimes et al. 2006). Although we are not able to add a thermal component to our fit, we find that the best fit power law hardly changes when we fit the $1-8 \mathrm{keV}$ band, $\Gamma_{1-8}=1.89 \pm 0.21$. Additionally, we measured that the contribution of the thermal component to the $2-10 \mathrm{keV}$ emission is $<3 \%$, using the fit from the X-ray spectrum for Haro 11 (Grimes et al. 2006). Furthermore, the thermal contribution is negligible $(<0.04 \%)$ in typical star-forming galaxies, based on the ratio of the hot gas component (Mineo et al. 2012b) to that from point sources (M12).

In their Figure 16, Xue et al. (2011) study X-ray sources from the $4 \mathrm{Ms}$ Chandra Deep Field-South (CDFS) survey to show that AGNs are unlikely to have this combination of low $0.5-8 \mathrm{keV} X$-ray count rate $\left(<10^{-3}\right.$ count $\left.\mathrm{s}^{-1}\right)$ and soft X-ray spectra $(\Gamma>1.5)$. Therefore, it is unlikely that all six LBAs harbor unobscured low-luminosity AGNs.

Based on the separate tests for the presence of AGNs discussed in this section, we conclude that it is unlikely that AGNs provide significant contributions to the $2-10 \mathrm{keV}$ emission for the LBAs in our sample.

\section{DISCUSSION}

Given the unlikely explanation that AGNs are causing the elevated $L_{\mathrm{X}} / \mathrm{SFR}$ in the LBAs, we focus on the XRB populations within these galaxies to address the question-could differences in either the XRB environments (i.e., due to effects that elevate $L_{\mathrm{X}} / \mathrm{SFR}$ at lower metallicities) or observing conditions (i.e., less dust attenuation absorbing the X-ray emission), between the LBAs and typical star-forming galaxies in the local universe, cause the $\sim 1.5 \sigma$ elevated $L_{\mathrm{X}} / \mathrm{SFR}$ ratios that are observed in LBAs? First, we discuss how $L_{X} / S F R$ relates to the XRB population within galaxies.

\subsection{HMXBs in Star-forming Galaxies}

In Figure 5, we show the relationship between $L_{X} / S F R$ and specific SFR $\left(\mathrm{sSFR}=\mathrm{SFR} / M_{\star}\right)$ for the LBAs (black points), stacked $z=1.5-4$ LBGs (blue squares) and comparison sample (gray symbols). LBAs, LBGs, and LIRGs/ULIRGs (gray triangles) have similarly high sSFRs, but $L_{X} / S F R$ is $\sim 1.5 \sigma$ higher for the LBAs, when compared to the fit and scatter of $L_{\mathrm{X}} / \mathrm{SFR}$ for HMXB-dominated galaxies (M12, gray dashed line/shaded region; see Equation (3)), suggesting that the XRB population in LBAs may be unique, even compared to other galaxies that have high sSFRs (see Section 4.2).

Within the XRB population, HMXBs are short-lived, tracing recent star formation activity (on timescales $\sim 10^{6-7} \mathrm{yr}$ ), while low-mass X-ray binaries (LMXBs) trace older stellar populations (for timescales $>10^{8-9} \mathrm{yr}$ ) and the $2-10 \mathrm{keV}$ X-ray luminosity scales with the stellar mass $\left(M_{\star}\right)$ of the galaxy. Assuming the following analytic parameterization for local (within a 
distance of $60 \mathrm{Mpc}$ ) normal galaxies

$$
\begin{gathered}
L_{\mathrm{X}}=L_{\mathrm{X}}(\mathrm{LMXB})+L_{\mathrm{X}}(\mathrm{HMXB}) \\
=\alpha M_{\star}+\beta \mathrm{SFR}
\end{gathered}
$$

L10 measure constants of $\alpha=(9.05 \pm 0.37) \times 10^{28} \mathrm{erg} \mathrm{s}^{-1} M_{\odot}^{-1}$ and $\beta=(1.62 \pm 0.22) \times 10^{39} \mathrm{erg} \mathrm{s}^{-1}\left(M_{\odot} \mathrm{yr}^{-1}\right)^{-1}$ (see also Colbert et al. 2004). Rearranging Equation (5) to give $L_{\mathrm{X}} / \mathrm{SFR}$ in terms of $\mathrm{SSFR}\left(\mathrm{SFR} / \mathrm{M}_{\star}\right) \mathrm{L} 10$ show that $\log$ $L_{\mathrm{X}} / \mathrm{SFR}$ is proportional to $\log (1 / \mathrm{sSFR}+$ constant $)$, causing two regimes: at low sSFRs $\left(\leqslant 10^{-10} \mathrm{yr}^{-1}\right)$, where both LMXBs and HMXBs contribute significantly to the X-ray luminosity, the relation is inversely linear; and at high sSFRs $\left(\geqslant 10^{-10} \mathrm{yr}^{-1}\right)$, where the contribution of LMXBs is negligible and the X-ray luminosity scales with SFR, the relation flattens (see black curve in Figure 5).

M12 correct the observed $0.5-2 \mathrm{keV}$ luminosities for contributions from LMXBs and hot gas in their sample of star-forming galaxies (gray points), and fit for the HMXB-driven $L_{X} / \mathrm{SFR}$ relation. After converting their relation to $2-10 \mathrm{keV}$, using $\Gamma=1.9$, we show the HMXB-driven X-ray/SFR relation by the gray dashed line (shaded region marks the $1 \sigma$ scatter) in Figures 2, 5, and 7. Hereafter, we refer to the relation as the local $L_{\mathrm{X}} / \mathrm{SFR}$ relation and restrict our analysis to high SSFR, HMXB-dominated galaxies, with sSFRs $>10^{-10} \mathrm{yr}^{-1}$ (right of the dotted line in Figure 5), to simplify the discussion by focusing on the relationship between galaxy properties and the HMXB population.

We note that most of the galaxies that appear to have elevated $(>1 \sigma) L_{X} /$ SFR in the low SFR end of Figure 2 have low sSFRs (LMXB-dominated; see Figure 5), where $L_{X} / \mathrm{SFR}$ is higher than for HMXB-dominated galaxies. In addition to the LBAs and stacked LBGs, Figure 5 reveals other HMXB-dominated galaxies with elevated $L_{X} / S F R$. These are discussed in more detail in the following section.

\subsection{Metallicity Dependence}

We return to the question whether metallicity differences between the LBA sample and other HMXB-dominated galaxies might drive the elevated $L_{X} / S F R$. In Figure 6 we show $L_{X} / S F R$ versus gas-phase metallicity for the LBAs (black points) and the partial comparison sample (symbols are described in legend of Figure 2). The data are correlated at the $99.2 \%$ significance level, based on a Spearman's rank correlation test. Optical spectral data are available for the full LBA sample, but only $40 \%$ of the HMXB-dominated (with sSFRs $>10^{-10} \mathrm{yr}^{-1}$ ) comparison sample.

Based on XRB population synthesis models, the formation of HMXB populations is strongly metallicity-dependent (e.g., Dray 2006; Linden et al. 2010; Fragos et al. 2013a, 2013b). One explanation is that stellar winds are weaker in massive stars with lower metallicities, allowing them to retain most of their mass and end their evolution as black hole companions. Since black hole HMXBs can be more luminous than neutron star HMXBs, the luminosity of an HMXB per SFR is expected to be higher in low-metallicity environments (Mapelli et al. 2009; Zampieri \& Roberts 2009; Fragos et al. 2013b). In addition, a reduced stellar wind would affect the secular evolution of the binary and would result in less orbital expansion due to angular momentum loss from the stellar wind, and in overall more systems that will encounter Roche-lobe overflow mass

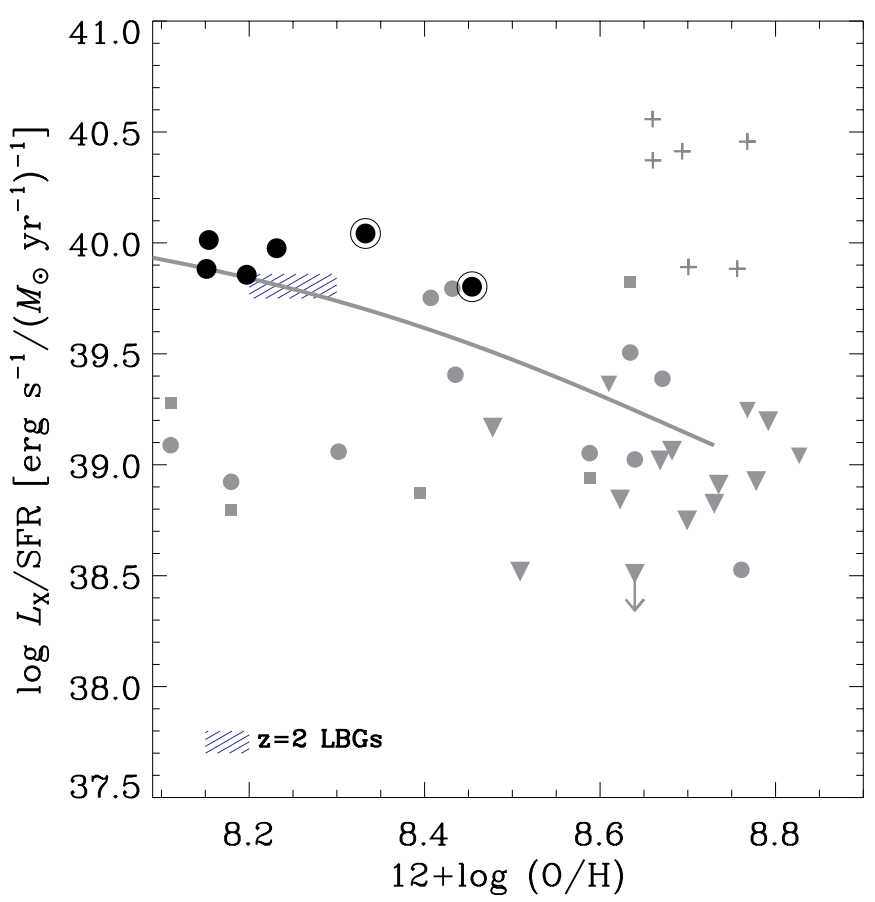

Figure 6. $L_{X} /$ SFR vs. gas-phase metallicity for galaxies with sSFR > $10^{-10} \mathrm{yr}^{-1}$, whose X-ray luminosities are expected to be dominated by HMXBs. The symbols are the same as in Figure 2, with the addition of a blue hatched region, corresponding to $z \sim 2$ LBGs (see the text for details). There is a correlation between $L_{X} /$ SFR and gas-phase metallicity $(12+\log [\mathrm{O} / \mathrm{H}])$, as predicted for HMXBs based on XRB population synthesis models (gray curve; Fragos et al. 2013b).

(A color version of this figure is available in the online journal.)

transfer instead of Bondi-Hoyle type wind accretion. Rochelobe overflow systems can drive much higher accretion rates onto the black hole and hence be more luminous X-ray sources (Fragos et al. 2013a). Indeed, initial results have shown evidence for an increased number of HMXBs, or off-nucleus ULXs, per SFR in lower-metallicity environments (Swartz et al. 2008; Mapelli et al. 2009, 2010, 2011; Kaaret et al. 2011; Prestwich et al. 2013).

While the lower metallicities in the LBA sample are not as extreme as in dwarf galaxies, Overzier et al. (2010) find that $z \sim 0.2$ LBAs have lower metallicities per stellar mass compared to the local mass-metallicity relation (e.g., Tremonti et al. 2004), but consistent to the mass-metallicity relation of LBGs at $z \sim 2$ (Erb et al. 2006). Additionally, Neill et al. (2011) link LBAs with the host galaxies of luminous supernovae, concluding that their low metallicities and high sSFRs may be related to extremely massive progenitors $\left(>100 M_{\odot}\right)$ or winddriven mass loss that may result in luminous supernovae.

We estimate metallicities for the $z=1.9$ and $2.5 \mathrm{LBG}$ samples from the X-ray stacking analysis in Basu-Zych et al. (2013) using the $z \sim 2$ mass-metallicity relation (Erb et al. 2006; converting the metallicities to PP04 O3N2 units, using the conversions given in Kewley \& Ellison 2008). The range of $L_{X} /$ SFR versus metallicity values for $z \sim 2$ LBGs is shown as a blue hatched region in Figure 6 . Based on the theoretical predictions that metallicity affects the number of luminous HMXBs, we postulate that the higher X-ray luminosity per SFR in the LBAs may be attributed to a higher number of more luminous HMXBs.

In addition to the LBAs and stacked LBGs, we note that several other HMXB-dominated galaxies appear to have elevated 

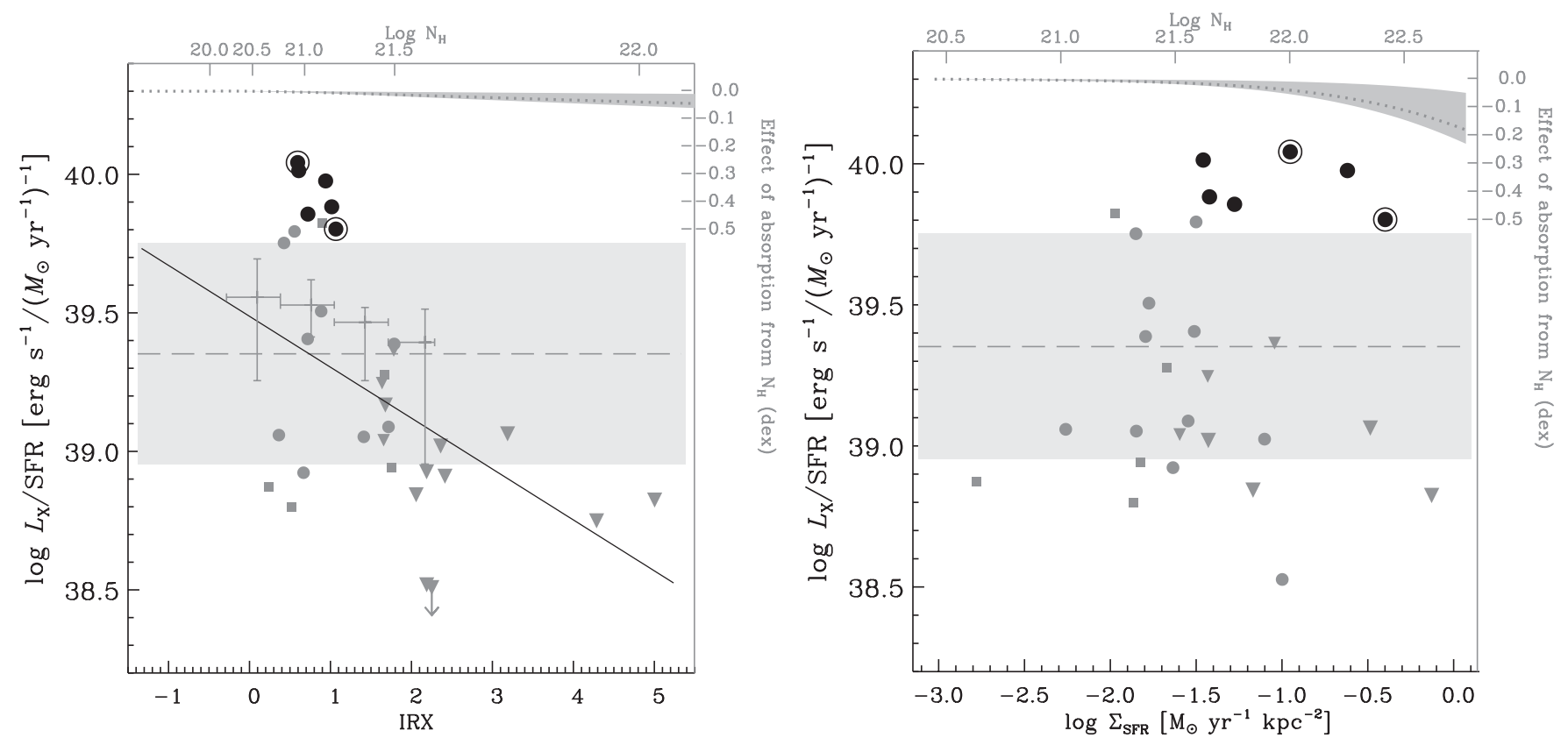

Figure 7. Using IRX (left panel) and SFR surface densities $\left(\Sigma_{\mathrm{SFR}}\right.$, right panel) to estimate the amount of neutral hydrogen (top axis), we find that X-ray absorption has a negligible effect on $L_{\mathrm{X}} / \mathrm{SFR}$ (dark gray region, with dotted gray curve corresponding to solar metallicity; right axis shows the effect of $N_{\mathrm{H}}$ on $L_{\mathrm{X}} / \mathrm{SFR}$ in dex units). Left: while the estimated $N_{\mathrm{H}}$ based on IRX shows negligible effect on $L_{\mathrm{X}} / \mathrm{SFR}, L_{\mathrm{X}} / \mathrm{SFR}$ does appear to correlate slightly (at $86 \%$ level) with IRX (solid black line). However, this is driven by the ULIRGs (see discussion in Section 4.3). For additional comparison, the gray error bars show the average values from M12 (see their Figure 11(d)), which appears consistent with our fit. Right: the SFR surface density (SFR/kpc ${ }^{-2}$ ) relates to the gas density, using the Schmidt-Kennicutt law. As expected from the estimated $N_{\mathrm{H}}$ (dark gray dotted line and region), $L_{\mathrm{X}} / \mathrm{SFR}$ is not affected significantly by $\Sigma_{\mathrm{SFR}}$, consistent with the observed lack of correlation.

$L_{\mathrm{X}} / \mathrm{SFR}>1 \sigma$ from the local $L_{\mathrm{X}} / \mathrm{SFR}$ relation (see Figure 5). We have metallicity estimates for two of these cases (NGC 3310 and NGC 2139), which have relatively low metallicities and appear above the gray solid line in Figure 6. In the other cases, low stellar masses $\left(<10^{10} M_{\odot}\right)$ imply that the expected metallicities are also low based on the mass-metallicity relation; some of these galaxies are well-known hosts to multiple ULXs, potentially attributed to their low metallicities (e.g., Cartwheel, Mapelli et al. 2009; NGC 1313, Zampieri \& Roberts 2009).

The gray line in Figure 6 shows the theoretical prediction, based on XRB population synthesis models (Fragos et al. 2013b). It is notable that these models predict that the X-ray luminosity from HMXBs per unit of SFR increases by approximately an order of magnitude going from solar metallicity to less than $10 \%$ solar. While theoretical models express metallicity in absolute $Z$ units, we convert these to units of oxygen abundance $(12+\log [\mathrm{O} / \mathrm{H}])$ by scaling solar metallicity $\left(Z_{\odot}\right)$ to $12+\log$ $[\mathrm{O} / \mathrm{H}] \sim 8.69$. Our analysis shows reasonable agreement with the theoretical prediction (gray curve), albeit with significant scatter, which is likely attributed to the issues discussed below.

We mention a couple of caveats to this analysis. First, we expect that the metallicities, measured using observed emission lines, are characteristic of star-forming regions where these lines are produced and HMXBs are presumed to be present. However, we are measuring the galaxy-averaged metallicity rather than the metallicities for the regions directly associated with HMXBs. The second caveat is that there is significant scatter between different techniques that measure metallicity and the absolute scaling is not well constrained (see, e.g., Kewley \& Ellison 2008 and the discussion in Section 2.4), which complicates comparisons with theoretical models. Nevertheless, since the former issue adds scatter via stochastic variations between the galaxies and the latter introduces systematic errors, the observed correlation shows promising evidence that metallicity may be responsible for driving much of the scatter in the $L_{\mathrm{X}} / \mathrm{SFR}$ relation, specifically offering a plausible explanation for the elevated $L_{\mathrm{X}} / \mathrm{SFR}$ in the lower-metallicity LBAs.

In Figure 3, the images of extended X-ray emission in three LBAs (VV 114, Haro 11, and J082355.0+280621.8) display multiple off-nuclear point sources. Given the X-ray sensitivity limit of the data, detected sources (ULXs) have $L_{X}>$ $10^{40} \mathrm{erg} \mathrm{s}^{-1}$ for VV 114 and Haro 11 and $>10^{41} \mathrm{erg} \mathrm{s}^{-1}$ for J082355.0+280621.8. Based on their SFRs and the analysis of HMXBs in star-forming galaxies by M12, at these limiting luminosities, we would have expected one or fewer detected ULXs in each galaxy compared to what we observe (five, three, and three off-nuclear sources in VV 114, Haro 11, and J082355.0+280621.8, respectively). Accounting for possible source confusion, where multiple sub-ULX sources appear as a single, unresolved $L_{\mathrm{X}}>10^{40} \mathrm{erg} \mathrm{s}^{-1}$ ULX, implies an improbably high number density of HMXBs per SFR. While further analysis is required to confirm this result, the numbers of ULXs suggest that LBAs host an excess of extremely luminous ULXs, potentially because they have uniquely high SFRs and low metallicities.

\subsection{Effects of Dust Extinction}

Typically, local galaxies appear to have higher levels of dust attenuation with increasing SFR (Wang \& Heckman 1996; Martin et al. 2005; Johnson et al. 2007), but the LBA sample appears to be a unique population that is offset toward lower IRX values per SFR (Basu-Zych et al. 2007; Overzier et al. 2011). We explore the possibility that LBAs appear to have elevated X-ray luminosities per SFR caused by dust effects. Since dust attenuation is uniquely low in LBAs, we might expect that typical galaxies suffer higher levels of dust obscuration, which might affect the observed $L_{X} / S F R$, i.e., if X-ray emission were significantly absorbed in other star-forming galaxies, such as IR-selected samples with similar SFRs. In Figure 7, we show our results for testing the hypothesis that $L_{\mathrm{X}} / \mathrm{SFR}$ depends on the 
amount of dust and gas, measured using two different methods: IRX (see Equation (2)) to measure the dust attenuation (shown in left panel), and the SFR surface density, $\Sigma_{\mathrm{SFR}}$, which is related to the gas surface density (Kennicutt 1998; henceforth referred to as the Schmidt-Kennicutt law, shown in the right panel).

In the left panel of Figure 7, we find that there is a weak correlation between $L_{X} /$ SFR and IRX, at an $86.4 \%$ confidence level, if we restrict our analysis to the HMXB-dominated (with sSFR $>10^{-10} \mathrm{yr}^{-1}$ ) galaxies with measured metallicities (the partial comparison sample, 38 galaxies, and 6 LBAs). The correlation is much stronger $(99.0 \%)$ if we include all of the HMXB-dominated galaxies (i.e., including galaxies without measured metallicities, 76 galaxies from the comparison sample and 6 LBAs). The gray error bars show the average values (in bins of IRX) from M12, which appear consistent with our fit (black line). Since dust and metallicity are also correlated in our data (at $~ 95 \%$ confidence level; see also Skibba et al. 2011), we consider the expected impact that dust extinction alone, using IRX as a proxy, might have on $L_{X} / S F R$. We estimate the neutral hydrogen column density from IRX using the following steps: (1) we convert IRX into the FUV attenuation $\left(A_{\mathrm{FUV}}\right)$ following the relation given by Equation (15) in Hao et al. (2011): $A_{\mathrm{FUV}}=2.5 \log \left[1+0.46 \times 10^{\mathrm{IRX}}\right] ;(2)$ we estimate the attenuation in the $V$-band from $A_{\mathrm{FUV}}$ as $A_{V} \approx 0.4 A_{\mathrm{FUV}}$, using the extinction laws from Gil de Paz et al. (2007): $A_{\mathrm{FUV}}=7.9 E(B-V)$ and Cardelli et al. (1989): $A_{V}=R_{V} E(B-V)$, where the $V$-band reddening is $R_{V}=3.1$; and (3) according to Güver \& Özel (2009), the neutral hydrogen column density $\left(N_{\mathrm{H}}\right)$ relates to the $V$-band attenuation as $N_{\mathrm{H}}=2.21 \times 10^{21} A_{V} \mathrm{~cm}^{-2}$. Based on these steps, we derive a relation between $N_{\mathrm{H}}$ and IRX as

$$
N_{\mathrm{H}} \approx 2.21 \times 10^{21}\left[0.98 \log \left(1+0.46 \times 10^{\mathrm{IRX}}\right)\right] \mathrm{cm}^{-2} .
$$

The top axis in Figure 7 (left panel) displays the corresponding neutral hydrogen column density and the gray dotted curve marks the nearly negligible effect $(<0.05$ dex, see right axis) of this X-ray absorption on $L_{X} / S F R$. Since the X-ray absorption is a product of the metallicity and hydrogen column density (our dotted curve assumes solar metallicity), the same values of $N_{\mathrm{H}}$ have a stronger effect on the level of X-ray absorption with increasing metallicity. The dark gray region shows the range of X-ray absorption expected given the observed range of metallicities in our samples, $12+\log [\mathrm{O} / \mathrm{H}]=8.1$ (top edge) -8.85 (bottom edge).

Since IRX provides a global measure of the dust attenuation averaged over the full extent of the galaxy, by comparing the IR emission to the UV emission, the IRX value may not accurately capture the true extinction present along the lines-of-sight to specific star-forming regions where HMXBs reside. Therefore, we try another independent measure to quantify the extinction in these galaxies.

The SFR surface density $\left(\mathrm{SFR} / \mathrm{kpc}^{2}, \Sigma_{\mathrm{SFR}}=\mathrm{SFR} / \pi a b\right.$, where $a$ and $b$ are the semi-major and semi-minor axes), is related to neutral gas surface density $\left(\Sigma_{\mathrm{H}}\right)$ as $\Sigma_{\mathrm{SFR}}=2.5 \times$ $10^{-4}\left[\Sigma_{\mathrm{H}} / M_{\odot} \mathrm{pc}^{-2}\right]^{1.4} M_{\odot} \mathrm{yr}^{-1} \mathrm{kpc}^{-2}$ (Kennicutt 1998). The neutral hydrogen gas column density is $\Sigma_{\mathrm{H}} / m_{\mathrm{H}}$, where $m_{\mathrm{H}}$ is the mass of a hydrogen atom. Therefore, we estimate $N_{\mathrm{H}}$ from the UV+IR-derived SFR surface density by

$$
N_{\mathrm{H}}=4.6 \times 10^{22} \Sigma_{\mathrm{SFR}}^{0.7} \mathrm{~cm}^{-2} .
$$

We see no correlation of $L_{\mathrm{X}} / \mathrm{SFR}$ with $\Sigma_{\mathrm{SFR}}$ (see right panel of Figure 7), as expected based on the small effect of $N_{\mathrm{H}}$ (derived from $\Sigma_{\mathrm{SFR}}$, as given in Equation (7); gray dotted curve) on
$L_{\mathrm{X}} / \mathrm{SFR}$. However, the Schmidt-Kennicutt law may not apply to galaxies with high SFR efficiencies, i.e., galaxies with high SFR surface density per gas density (e.g., LBAs, Basu-Zych 2009; and possibly LIRGS, García-Burillo et al. 2012), which explains why the estimated $N_{\mathrm{H}}$ values from the two techniques differ. However, the two techniques do provide comparable values of $N_{\mathrm{H}}$ for the other star-forming galaxies (gray squares and points in Figure 7; correlated at $85 \%$ confidence level according to a Spearman's rank correlation test).

Neither measure of extinction implies sufficient $N_{\mathrm{H}}$ to significantly absorb 2-10 keV X-ray emission. However, since both IRX and $\Sigma_{\text {SFR }}$ are calculated using global properties of the galaxies, we are measuring the average absorption and are unable to account for heavily obscured sub-galactic regions. For example, in ULIRGs, Genzel et al. (1998) find incredibly high optical absorption values, $A_{V} \approx 50-1000$ mag for some ULIRGs, where the optical dust column densities are comparable to the dense molecular cloud (CO) millimeter observations. Therefore, HMXBs found in such heavily obscured regions would be affected by significant X-ray absorption and in such specific cases the observed X-ray luminosities may be underestimated. While this scenario is plausible in some ULIRGs and LIRGs, $\mathrm{X}$-ray absorption is not likely to fully explain the apparent correlation between $L_{X} / S F R$ and IRX across the entire population of star-forming galaxies. However, the correlation between $L_{X} /$ SFR and IRX is mainly influenced by the ULIRGs; i.e., the data are not correlated ( $<50 \%$ significance level) when ULIRGs are eliminated.

\section{IMPLICATIONS FOR X-RAY EMISSION IN HIGH REDSHIFT GALAXIES}

While in this study we are studying the X-ray emission in low redshift $(z<0.1)$ analogs of LBGs, a parallel aim is to gain some physical insight about the important factors that affect X-ray emission in distant LBGs. Since individual X-ray detections of LBGs at $z>1$ are likely dominated by AGNs, the study of XRB populations in these galaxies is limited to their average X-ray emission based on stacking of large numbers of galaxies. Basu-Zych et al. (2013) perform stacking of $z=1.5-5$ LBGs in the 4 Ms CDFS, carefully eliminating sources that were individually detected (potential AGNs) and correcting for any AGN contamination by subtracting the expected AGN fraction, using the local AGN luminosity function to estimate the AGN fraction. Therefore, the LBG stacking results from this study present the X-ray/SFR relation for $z=1.5-5$ starforming galaxies and find evolution in the $L_{X} / S F R$ ratio over the history of the universe as described by the following equation: $\log L_{\mathrm{X}}=0.90 \log (1+z)+0.65 \log \mathrm{SFR}+39.8$. Fragos et al. (2013a), combining XRB population synthesis models with results from the Millennium II simulation and semi-analytical galaxy catalogs (Guo et al. 2011), similarly predict that the X-ray emission from HMXBs per unit SFR evolves with redshift, driven by the redshift evolution in the mean stellar metallicity. In this paper, we arrive at the same conclusion using individual LBAs.

Based on the numbers of resolved HMXBs within a few extended LBAs, we find additional clues that these lower-metallicity, high SFR galaxies contain a higher frequency of ULXs with $L_{X}>10^{40} \mathrm{erg} \mathrm{s}^{-1}$. Investigating the luminosity distributions of HMXBs in LBGs is impossible, due to the high spatial resolution and sensitivity requirements. However, extrapolating our result to higher redshift LBGs, we might expect that galaxies in the early universe hosted a larger number 
of ULXs than predicted based on typical star-forming galaxies (e.g., M12).

\section{SUMMARY AND FUTURE WORK}

In this paper, we present our results on the X-ray emission from six LBAs in the local universe $(z<0.1)$. Whereas our knowledge about the high energy emission from star-forming galaxies in the early universe $(z \gtrsim 1)$ is limited by statistical techniques such as stacking, in this paper, we study the $\mathrm{X}$-ray emission in individual galaxies from a sample of $z<0.1$ galaxies that are excellent analogs to distant LBGs. Based on our comparison of LBAs with other star-forming galaxies, we summarize our main results as follows.

1. We find that the hard $(2-10 \mathrm{keV}) \mathrm{X}$-ray emission per unit SFR is elevated by $\sim 1.5 \sigma$ in the LBA population, similar to X-ray stacking results from $z>3$ LBGs (Figure 2).

2. We find that the lower metallicities in the LBA sample may be driving the elevated $L_{X} /$ SFR (Figure 6).

3. We also find that this effect is not driven by extinction as we find no significant correlation of $L_{X} / S F R$ with IRX or SFR surface density and the inferred column densities are too low to cause significant absorption in the 2-10 keV X-ray luminosity (Figure 7).

Our study of X-ray emission in LBAs, a unique population of galaxies having high SFR and low metallicities, highlights the importance of metallicity on the scaling between HMXB $\mathrm{X}$-ray emission and SFR. However, this result is limited by the small sample size; a sample spanning a wider range of SFRs, metallicities, and dust attenuation values would better constrain the physical properties responsible for driving the elevated $L_{\mathrm{X}} / \mathrm{SFR}$ observed in the current LBA sample.

We find tentative evidence that LBAs, possibly owing to their high SFRs and low metallicities, host a significant excess of $>10^{40} \mathrm{erg} \mathrm{s}^{-1}$ ULXs. This result is based on Chandra observations of individually resolved $X$-ray sources within two extended LBAs, VV114 and J082355.0+280621.8. However, the data for J082355.0+280621.8 were not complete at the $10^{40} \mathrm{erg} \mathrm{s}^{-1}$ limit and require deeper follow-up observations. Additional observations of spatially extended LBAs, only possible with the high angular resolution capabilities of Chandra, would provide better statistics on the distribution of XRB populations, in order to study the high-luminosity tail of the X-ray luminosity function (XLF) which can be compared to XLFs of local star-forming galaxies (e.g., M12) and X-ray population synthesis models (see, e.g., Tzanavaris et al. 2013).

While the ability to study XRB populations in individual high redshift $(z>2)$ LBGs may come to fruition in the future (e.g., an X-Ray Telescope with large collecting area such as $S M A R T-\mathrm{X})$, these $z<0.1$ analogs offer some important clues suggesting that the relationship between $L_{X}$ and SFR evolves with the chemical evolution of the universe. In this paper, we establish that such a relationship is not merely occurring in an average sense over cosmic time (Basu-Zych et al. 2013; Fragos et al. 2013a), but that the scatter in the $L_{X} /$ SFR relation may be attributed to metallicity variations between individual galaxies.

This research was supported by Chandra Cycle 12 program No. 12620841 (PI: Basu-Zych). We thank our anonymous referee for helpful suggestions, A. Prestwich for sharing her work on ULXs in extremely low metallicity galaxies, S. Mineo for providing useful comparison data, and J. Mullaney for sharing his IR code. The author gratefully acknowledges B.
D. Johnson, A. Henry, and V. Antoniou for helpful discussions. T.F. acknowledges support from CfA and ITC prize fellowship programs. This publication makes use of data products from the Wide-field Infrared Survey Explorer, which is a joint project of the University of California, Los Angeles, and the Jet Propulsion Laboratory/California Institute of Technology, funded by the National Aeronautics and Space Administration.

\section{REFERENCES}

Adelberger, K. L., \& Steidel, C. C. 2000, ApJ, 544, 218

Alexandroff, R., Overzier, R. A., Paragi, Z., et al. 2012, MNRAS, 423, 1325

Baldwin, J. A., Phillips, M. M., \& Terlevich, R. 1981, PASP, 93, 5

Basu-Zych, A. R. 2009, PhD Thesis, Columbia Univ.

Basu-Zych, A. R., Gonçalves, T. S., Overzier, R., et al. 2009, ApJL, 699, L118 Basu-Zych, A. R., Lehmer, B. D., Hornschemeier, A. E., et al. 2013, ApJ, 762,45

Basu-Zych, A. R., Schiminovich, D., Johnson, B. D., et al. 2007, ApJS, 173,457

Bauer, F. E., Alexander, D. M., Brandt, W. N., et al. 2002, AJ, 124, 2351

Bell, E. F., \& de Jong, R. S. 2001, ApJ, 550, 212

Bell, E. F., McIntosh, D. H., Katz, N., \& Weinberg, M. D. 2003, ApJS, 149, 289 Bell, E. F., Papovich, C., Wolf, C., et al. 2005, ApJ, 625, 23

Bergvall, N., \& Östlin, G. 2002, A\&A, 390, 891

Blanton, M. R., Brinkmann, J., Csabai, I., et al. 2003, AJ, 125, 2348

Brandt, W. N., Hornschemeier, A. E., Schneider, D. P., et al. 2001, ApJL, 558, L5

Cardelli, J. A., Clayton, G. C., \& Mathis, J. S. 1989, ApJ, 345, 245

Chary, R., \& Elbaz, D. 2001, ApJ, 556, 562

Colbert, E. J. M., Heckman, T. M., Ptak, A. F., Strickland, D. K., \& Weaver, K. A. 2004, ApJ, 602, 231

Cormier, D., Lebouteiller, V., Madden, S. C., et al. 2012, A\&A, 548, A20

Cowie, L. L., Barger, A. J., \& Hasinger, G. 2012, ApJ, 748, 50

de Vaucouleurs, G., de Vaucouleurs, A., Corwin, H. G., Jr., et al. 1991, Third Reference Catalogue of Bright Galaxies. Vol. I: Explanations and References. Vol. II: Data for Galaxies Between $0^{h}$ and $12^{h}$. Vol. III: Data for Galaxies Between $12^{h}$ and $24^{h}$ (New York: Springer)

Dray, L. M. 2006, MNRAS, 370, 2079

Erb, D. K., Shapley, A. E., Pettini, M., et al. 2006, ApJ, 644, 813

Fabbiano, G. 1989, ARA\&A, 27, 87

Fragos, T., Lehmer, B., Tremmel, M., et al. 2013a, ApJ, 764, 41

Fragos, T., Lehmer, B. D., Naoz, S., Zezas, A., \& Basu-Zych, A. R. 2013b, arXiv:1306.1405

García-Burillo, S., Usero, A., Alonso-Herrero, A., et al. 2012, A\&A, 539, A8

Genzel, R., Lutz, D., Sturm, E., et al. 1998, ApJ, 498, 579

Gil de Paz, A., Boissier, S., Madore, B. F., et al. 2007, ApJS, 173, 185

Gilfanov, M. 2004, MNRAS, 349, 146

Gonçalves, T. S., Basu-Zych, A., Overzier, R., et al. 2010, ApJ, 724, 1373

Grimes, J. P., Heckman, T., Hoopes, C., et al. 2006, ApJ, 648, 310

Grimes, J. P., Heckman, T., Strickland, D., \& Ptak, A. 2005, ApJ, 628, 187

Grimes, J. P., Heckman, T., Strickland, D., et al. 2007, ApJ, 668, 891

Grimm, H.-J., Gilfanov, M., \& Sunyaev, R. 2003, MNRAS, 339, 793

Guo, Q., White, S., Boylan-Kolchin, M., et al. 2011, MNRAS, 413, 101

Güver, T., \& Özel, F. 2009, MNRAS, 400, 2050

Hao, C.-N., Kennicutt, R. C., Johnson, B. D., et al. 2011, ApJ, 741, 124

Heckman, T. M., Borthakur, S., Overzier, R., et al. 2011, ApJ, 730, 5

Heckman, T. M., Hoopes, C. G., Seibert, M., et al. 2005, ApJL, 619, L35

Heckman, T. M., Robert, C., Leitherer, C., Garnett, D. R., \& van der Rydt, F. 1998, ApJ, 503, 646

Hoopes, C. G., Heckman, T. M., Salim, S., et al. 2007, ApJS, 173, 441

Hornschemeier, A. E., Brandt, W. N., Garmire, G. P., et al. 2001, ApJ, 554, 742

Hornschemeier, A. E., Heckman, T. M., Ptak, A. F., Tremonti, C. A., \& Colbert, E. J. M. 2005, AJ, 129, 86

Howell, J. H., Armus, L., Mazzarella, J. M., et al. 2010, ApJ, 715, 572

Iwasawa, K., Sanders, D. B., Teng, S. H., et al. 2011, A\&A, 529, A106

Jarrett, T. H., Cohen, M., Masci, F., et al. 2011, ApJ, 735, 112

Jia, J., Ptak, A., Heckman, T. M., et al. 2011, ApJ, 731, 55 (J11)

Johnson, B. D., Schiminovich, D., Seibert, M., et al. 2007, ApJS, 173, 377

Kaaret, P., Schmitt, J., \& Gorski, M. 2011, ApJ, 741, 10

Kauffmann, G., Heckman, T. M., Tremonti, C., et al. 2003, MNRAS, 346, 1055

Kennicutt, R. C. 1998, ApJ, 498, 541

Kewley, L. J., Dopita, M. A., Sutherland, R. S., Heisler, C. A., \& Trevena, J. 2001, ApJ, 556, 121

Kewley, L. J., \& Ellison, S. L. 2008, ApJ, 681, 1183 
Kim, D.-C., Sanders, D. B., Veilleux, S., Mazzarella, J. M., \& Soifer, B. T. 1995, ApJS, 98, 129

Kroupa, P. 2001, MNRAS, 322, 231

Laird, E. S., Nandra, K., Hobbs, A., \& Steidel, C. C. 2006, MNRAS, 373, 217

Lehmer, B. D., Alexander, D. M., Bauer, F. E., et al. 2010, ApJ, 724, 559 (L10)

Lehmer, B. D., Brandt, W. N., Alexander, D. M., et al. 2005, AJ, 129, 1

Lehmer, B. D., Brandt, W. N., Alexander, D. M., et al. 2008, ApJ, 681, 1163

Li, J.-T., \& Wang, Q. D. 2013, MNRAS, 428, 2085

Linden, T., Kalogera, V., Sepinsky, J. F., et al. 2010, ApJ, 725, 1984

Mapelli, M., Colpi, M., \& Zampieri, L. 2009, MNRAS, 395, L71

Mapelli, M., Ripamonti, E., Zampieri, L., \& Colpi, M. 2011, AN, 332, 414

Mapelli, M., Ripamonti, E., Zampieri, L., Colpi, M., \& Bressan, A. 2010, MNRAS, 408, 234

Martin, D. C., Seibert, M., Buat, V., et al. 2005, ApJL, 619, L59

Mineo, S., Gilfanov, M., \& Sunyaev, R. 2012a, MNRAS, 419, 2095

Mineo, S., Gilfanov, M., \& Sunyaev, R. 2012b, MNRAS, 426, 1870

Morrissey, P., Conrow, T., Barlow, T. A., et al. 2007, ApJS, 173, 682

Moustakas, J., \& Kennicutt, R. C., Jr. 2006, ApJS, 164, 81

Nandra, K., Mushotzky, R. F., Arnaud, K., et al. 2002, ApJ, 576, 625

Neill, J. D., Sullivan, M., Gal-Yam, A., et al. 2011, ApJ, 727, 15

Overzier, R. A., Heckman, T. M., Kauffmann, G., et al. 2008, ApJ, 677, 37

Overzier, R. A., Heckman, T. M., Schiminovich, D., et al. 2010, ApJ, 710, 979

Overzier, R. A., Heckman, T. M., Tremonti, C., et al. 2009, ApJ, 706, 203

Overzier, R. A., Heckman, T. M., Wang, J., et al. 2011, ApJL, 726, L7

Persic, M., \& Rephaeli, Y. 2007, A\&A, 463, 481
Pettini, M., \& Pagel, B. E. J. 2004, MNRAS, 348, L59

Prestwich, A. H., Tsantaki, M., Zezas, A., et al. 2013, ApJ, 769, 92

Ranalli, P., Comastri, A., \& Setti, G. 2003, A\&A, 399, 39

Seibert, M., Heckman, T. M., \& Meurer, G. R. 2002, AJ, 124, 46

Shemmer, O., Brandt, W. N., Netzer, H., Maiolino, R., \& Kaspi, S. 2006, ApJL, 646, L29

Skibba, R. A., Engelbracht, C. W., Dale, D., et al. 2011, ApJ, 738, 89

Strickland, D. K., Heckman, T. M., Weaver, K. A., \& Dahlem, M. 2000, AJ, 120,2965

Swartz, D. A., Ghosh, K. K., Tennant, A. F., \& Wu, K. 2004, ApJS, 154, 519

Swartz, D. A., Soria, R., \& Tennant, A. F. 2008, ApJ, 684, 282

Symeonidis, M., Georgakakis, A., Seymour, N., et al. 2011, MNRAS, 417, 2239

Tremonti, C. A., Heckman, T. M., Kauffmann, G., et al. 2004, ApJ, 613, 898

Trouille, L., Barger, A. J., \& Tremonti, C. 2011, ApJ, 742, 46

Tzanavaris, P., et al. 2013, submitted

Wang, B., \& Heckman, T. M. 1996, ApJ, 457, 645

Webb, T. M., Eales, S., Foucaud, S., et al. 2003, ApJ, 582, 6

Wright, E. L., Eisenhardt, P. R. M., Mainzer, A. K., et al. 2010, AJ, 140, 1868

Wu, H., Zou, Z. L., Xia, X. Y., \& Deng, Z. G. 1998, A\&AS, 132, 181

Xue, Y. Q., Luo, B., Brandt, W. N., et al. 2011, ApJS, 195, 10

Zampieri, L., \& Roberts, T. P. 2009, MNRAS, 400, 677

Zheng, Z.-Y., Malhotra, S., Wang, J.-X., et al. 2012, ApJ, 746, 28

Zibetti, S., Charlot, S., \& Rix, H.-W. 2009, MNRAS, 400, 1181

Zinn, P.-C., Blex, S., Seymour, N., \& Bomans, D. J. 2012, A\&A, 547, A50 